\title{
基于有机二硫化物氧化还原电对的非碘系染料敏化太阳能电池
}

\author{
马英壮郑灵灵 \\ 张立培 \\ 陈志坚 \\ 王树峰 \\ 曲波 \\ 肖立新* \\ 龚旗煌 \\ (北京大学物理学院 人工微结构和介观物理国家重点实验室＼cjkstart北京 100871)
}

\begin{abstract}
摘要 相比较于硅太阳能电池而言, 染料敏化太阳能电池具有成本低廉、制备简单的特点. 在目前研究中, 普遍采用碘 离子作为氧化还原电对, 然而碘元素本身具有腐蚀性, 长期使用会腐蚀对电极从而影响器件寿命, 而且此类电解液在 可见光区内有很强的吸收, 也不利于光能向电能的转换. 本文采用了一种新型有机二硫化物, 2,5-二硫基-1,3,4-噻二唑, 作为氧化还原介质，其不仅能够利用其自身自均聚/自解聚反应来实现光电转换过程，降低对电极的腐蚀，而且在可见 光区内的吸收很低, 也有利于制作背入射式的染料敏化太阳能电池体系. 通过器件的优化, 可以分别实现 $1.6 \%$ (100 $\left.\mathrm{mW} \cdot \mathrm{cm}^{-2}\right)$ 和 $2.6 \%\left(13 \mathrm{~mW} \cdot \mathrm{cm}^{-2}\right)$ 的光电转换效率.
\end{abstract}

关键词 染料敏化; 太阳能电池; 有机二硫化物; 氧化还原电对

\section{A Novel Organic Disulfide/Thiolate Redox Mediator for Iodine-free Dye-sensitized Solar Cells}

\author{
Ma, Yingzhuang Zheng, Lingling Zhang, Lipei Chen, Zhijian \\ Wang, Shufeng Qu, Bo Xiao, Lixin* Gong, Qihuang \\ (State Key Laboratory for Mesoscopic Physics and Department of Physics, Peking University, Beijing, 100871)
}

\begin{abstract}
Over the last 20 years, much attention has been paid to renewable energy technology. Photovoltaic is a promising alternative to conventional fossil fuels. Dye-sensitized solar cells (DSCs) attract notable interest, not only due to their high efficiency and environmentally friendly nature, but also their easy fabrication and relatively low manufacture costs. Despite the high efficiencies, iodine/triiodine electrolytes have some disadvantages, such as the corrosion of the metallic electrodes and the sealing materials. It also absorbs visible light around $430 \mathrm{~nm}$. Therefore, it is important to exploit the iodine-free redox couple in DSCs. An organic disulfide material of 2,5-dimercapto-1,3,4-thiadiazole (DMcT) is proved here to reduce and oxidize independently via homopolymerization and depolymerization. DMcT has been applied as cathode active material for lithium rechargeable batteries. Meanwhile, the self-redox property could be used as redox mediator in lieu of iodine/triiodine electrolytes. DMcT can be oxidized by self-polymerizing into PDMcT, which can be reduced by depolymerizing back to DMcT. In contrast to the conventional redox couples consisted of two different materials, DMcT can independently act as the redox mediator, which is the main difference between DMcT and the redox couples reported previously. Dye-sensitized solar cells consist of mesoporous $\mathrm{TiO}_{2}$, N719 dye, and this novel electrolyte achieved power conversion efficiency of $1.6 \%$ under $100 \mathrm{~mW} \cdot \mathrm{cm}^{-2}$ simulated sunlight (AM $\left.1.5 \mathrm{G}\right)$ and a higher efficiency of $2.6 \%$ at weak illumination $\left(13 \mathrm{~mW} \cdot \mathrm{cm}^{-2}\right)$, implying its promising application prospect. Although the conversion efficiency is relatively low to the iodine/triiodine-based DSCs, this novel single self-redox mediator provides a new promising way to the iodine-free dye-sensitized solar cells.

Keywords dye-sensitized; solar cell; organic disulfide; redox couple
\end{abstract}

\section{1 引言}

新型能源是人类社会迫切需要解决的最重要问题 之一, 可以解决矿物能源的枯竭以及使用中对环境的破 坏, 保障社会的可持续发展. 其中太阳能的收集和利用 是最具前景的方案. 太阳能的利用具有极高的灵活性, 从大规模的光能源电厂、家用太阳能发电, 到小型电子 设备, 都可以利用经由太阳光转换产生的能源. 因此,
如何实现高效的光电转换成为太阳能利用中的核心问 题之一. 染料敏化太阳能电池自问世以来，即以其低成 本、易制备、高效率、低污染等特点引起了广大专家学 者的高度关注. 近二十年来, 有关于染料敏化太阳能电 池的报道不断增加, 无论是新材料的合成, 还是新器件 结构的设计等方面都取得了很大的进展 ${ }^{[1>8]}$. 一般来说, 染料敏化太阳能电池由以下几个部分组成: 透明导电祄 底, 金属氧化物光阳极, 吸光染料, 氧化还原电解质,

*E-mail: 1xxiao@pku.edu.cn; Tel.: 010-62757290

Received September 24, 2014; published November 21, 2014.

Supporting information for this article is available free of charge via the Internet at http://sioc-journal.cn.

Project supported by the National Natural Science Foundation of China (Nos. 61177020, 11121091).

项目受国家自然科学基金(Nos. 61177020, 11121091)资助. 
以及对电极等共同组成. 当光子入射到染料时, 染料受 到激发后会向光阳极注入电子, 而失去电子的染料则通 过氧化还原电对获得电子回到基态, 而氧化还原电对则 通过对电极接受外电路的电子，从而完成整个电流回 路, 可以说氧化还原电对在染料敏化电池中起着 “承上 启下” 的关键作用. 在目前的研究中, 碘系氧化还原电 对 $\left(\mathrm{I}^{-} / \mathrm{I}_{3}{ }^{-}\right)$表现出了高效的电荷转移效率, 也是目前染料 敏化太阳能电池中应用最广泛的一类氧化还原电 对 ${ }^{[9]}$. 虽然碘系氧化还原电对表现出了较高的光电转化 效率, 但是其本身也有一定的缺陷, 限制了其进一步的 发展 ${ }^{[10,11]}$. 比如, 采用 $\mathrm{I}^{-} / \mathrm{I}_{3}{ }^{-}$的电解液在可见光区内有较 强的吸收, 这意味着入射光的能量无法被染料充分利 用, 此外碘本身具有一定的腐蚀性, 如果长期使用会影 响金属对电极的催化活性, 而且对于液态电解液的封装 材料也有较大影响, 造成漏液、挥发等结果, 不利于提 高器件寿命 ${ }^{[12]}$. 因此开发新型的非碘系氧化还原电对 是一个非常具有实际意义的课题 ${ }^{[13 ~ 17]}$. 近期, Wang 等 开发了基于有机二硫化物/硫醇盐的氧化还原电对 (5,5'-dithiobis(1-methyltetrazole)/1-methyltrazole-5thiolate, $\mathrm{T}^{-} / \mathrm{T}_{2}$ ), 利用 $\mathrm{S}-\mathrm{S}$ 键的结合与断开的氧化还原 反应完成电荷转移 ${ }^{[13]}$. 类似的, 其他有机硫化物的氧化 还原电对, 2,2'-dithiobis(5-methyl-1,3,4-thiadiazole)/5methyl-1,3,4-thiadiazole-2-thiolate $\left(\mathrm{McMT}^{-} / \mathrm{BMT}\right)$ 以及 dithioabis-( $N, N, N^{\prime}, N^{\prime}$-tetramethylformamidinium)/ $N, N, N^{\prime}, N^{\prime}$-tetramethylthiourea (TMFDS $\left.{ }^{2+} / \mathrm{TMTU}\right)$ 等也相 继报道, 其中基于 $\mathrm{T}^{-} / \mathrm{T}_{2}$ 的染料敏化太阳能电池效率达

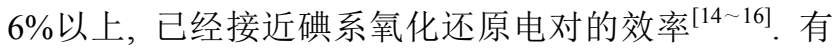
机硫化物氧化还原电对收到了广泛的关注, 主要是其对 入射光吸收较低, 而且腐蚀性较低, 有利于保证器件的 长期寿命.

本工作中采用了 2,5-Dimercapto-1,3,4-thiadiazole (DMcT) 作为氧化还原电解质, 利用其自均聚/自解聚的 过程, 即可独立完成氧化还原过程的电荷输运. 与采用 两种材料作为电对的方法不同, 只用 DMcT 自身一种材 料, 即可完成氧化还原反应. 采用这种方法制备氧化还 原电解质, 不仅能够避免碘系材料的缺点, 同时能够简 化器件制备过程, 并降低材料生产成本, 为开发非碘系 染料敏化太阳能电池提供了新的可能.

\section{2 结果与讨论}

Visco 等首先证实了 DMcT 可以进行自均聚/自解聚 反应, 并且将其利用到了锂离子电池的电极中 ${ }^{[19 \sim 21]}$. $\mathrm{DMcT}$ 的自均聚/自解聚反应过程如图 1 所示, DMcT 可 以通过聚合反应失去电子, 被氧化形成聚 DMcT; 反之, 聚 DMcT 可以通过解聚合反应得到电子, 被还原成为 $\mathrm{DMcT}$, 从而完成自氧化还原过程.

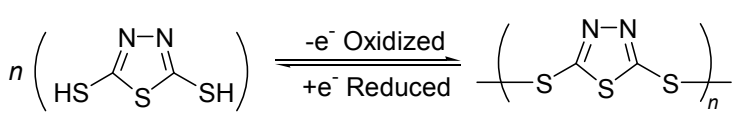

图 $1 \mathrm{DMcT}$ 的自均聚/自解聚反应

Figure 1 Homopolymerization and depolymerization of DMcT.

\section{1 器件的光伏特性}

在太阳光模拟器照射下, 测出器件伏安特性曲线, 结合器件的开路电压 $V_{\mathrm{oc}}$ 与短路电流 $J_{\mathrm{sc}}$, 可以计算出填 充因子 $F F$ 与能量转换效率 $\eta$,

$$
\begin{aligned}
& F F=\left(J_{\max } \times V_{\text {max }}\right) /\left(J_{\text {sc }} \times V_{\text {oc }}\right) \\
& \eta=\left(F F \times J_{\text {sc }} \times V_{\text {oc }}\right) / P_{\text {inc }}
\end{aligned}
$$

其中 $J_{\max }$ 与 $V_{\text {max }}$ 代表最大输出功率点处的电流值与电压 值, $P_{\mathrm{inc}}$ 为入射光的光功率, 相关数据如图 2 , 表 1 所示.

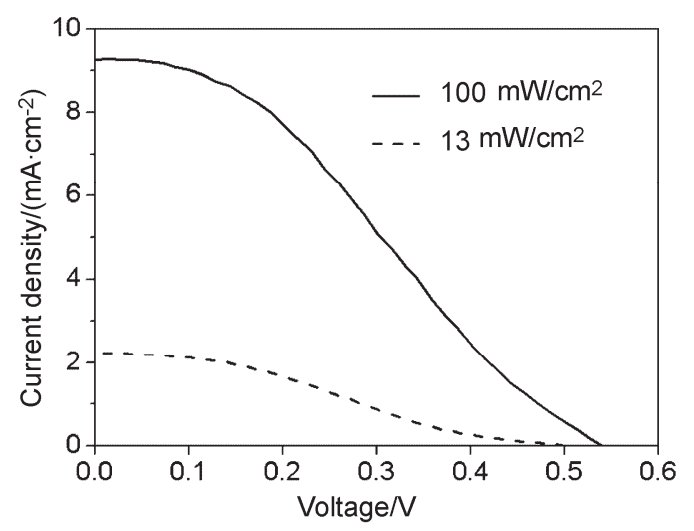

图 2 太阳能电池伏安特性曲线

Figure 2 Voltage-current curves of the solar cell

表 1 太阳能电池器件光伏特性

Table 1 Photovoltaic characteristics of the solar cell

\begin{tabular}{ccccc}
\hline 光强 $/\left(\mathrm{mW} \cdot \mathrm{cm}^{-2}\right)$ & $V_{\mathrm{oc}} / \mathrm{V}$ & $J_{\mathrm{sc}}\left(\mathrm{mA} \cdot \mathrm{cm}^{-2}\right)$ & $F F$ & $\eta / \%$ \\
\hline 100 & 0.54 & 9.3 & 0.3 & 1.6 \\
13 & 0.51 & 2.2 & 0.3 & 2.6 \\
\hline
\end{tabular}

测试数据表明在标准光强下, 采用 $\mathrm{DMcT}$ 电解液的 器件 $\mathrm{A}$ 的 $J_{\mathrm{sc}}$ 为 $9.3 \mathrm{~mA} \cdot \mathrm{cm}^{-2}, V_{\mathrm{oc}}$ 为 $0.54 \mathrm{~V}, F F$ 为 0.33 , 效率 $\eta$ 为 $1.6 \%$, 图 1 中实线代表了器件的伏安特性曲线, 可以看出, 导致器件最终效率不高的原因主要是其 $F F$ 还相对较低. 此外, 氧化还原电位直接决定了器件 $V_{\mathrm{oc}}$ 的大小, 通过循环伏安法测试发现, 与其他氧化还原电 对相比(如 $\mathrm{T}^{-} / \mathrm{T}_{2}$ ), 见图 $\mathrm{S} 1$, DMcT 的氧化还原电位与 $\mathrm{TiO}_{2}$ 的导带能级更加接近, 导致 $V_{\mathrm{oc}}$ 相对较低. 此外, DMcT 的氧化峰与还原峰的电势差相差更大, 与 $\mathrm{T}^{-} / \mathrm{T}_{2}$ 相比, 也不利于氧化还原反应的进行, 从而导致电荷内 部复合的加剧. 为了更充分的体现器件的光电转换特 性, 还测试了器件在弱光条件下的效率, 将入射光强减 小到 $13 \mathrm{~mW} \cdot \mathrm{cm}^{-2}$, 器件的 $J_{\mathrm{sc}}$ 为 $2.2 \mathrm{~mA} \cdot \mathrm{cm}^{-2}, V_{\mathrm{oc}}$ 为 $0.51 \mathrm{~V}, F F$ 为 0.30 , 效率 $\eta$ 为 $2.6 \%$. 可以发现, 虽然 $V_{\mathrm{oc}}$ 和 $F F$ 在弱光条件下略有下降, 但是器件在弱光下的光 电流仍然相对较高，最终效率达到 $2.6 \%$, 相比于标准光 强下的效率有明显的提高, 这说明器件在较弱的光照环 
境下有较好的表现, 在类似于室内等弱光照条件下有着 较好的应用前景.

\section{2 器件的光谱响应}

单色光电转化效率(incident photon-to-electron conversion efficiency, IPCE), 即入射单色光子-电子转化效 率，其表达式如公式(3)所示.

$$
\operatorname{IPCE}(\lambda)=(h c / e \lambda)\left(J_{\mathrm{sc}}(\lambda) / P_{\text {in }}(\lambda)\right)
$$

其中, $h$ 是普朗克常数, $c$ 是光速, $e$ 是基本电荷常数, $\lambda$ 是 入射光波长, $J_{\mathrm{sc}}(\lambda)$ 是入射光波长为 $\lambda$, 入射光功率为 $P_{\text {in }}(\lambda)$ 时, 接收到的光电流. 通过图 3 可以发现, 器件在 可见光区内对于入射光有较强的光响应，在 $400 \sim 550$ $\mathrm{nm}$ 之间的 IPCE 值在 50\%以上, 有利于充分利用入射光 能. 此外, 为了模拟实际器件中电解液的吸收, 采用与 制备器件相同的方式, 将 DMcT 电解液滴入两块 FTO 玻璃之间, 然后再测量吸收谱. 由图 3 可以发现, DMcT 电解液的吸收峰位于 $400 \mathrm{~nm}$ 之前，从而避开了 N719 染 料的光谱响应范围, 有效的避免了电解液与染料对于入 射光吸收的相互竞争, 从而保证染料分子的光吸收.

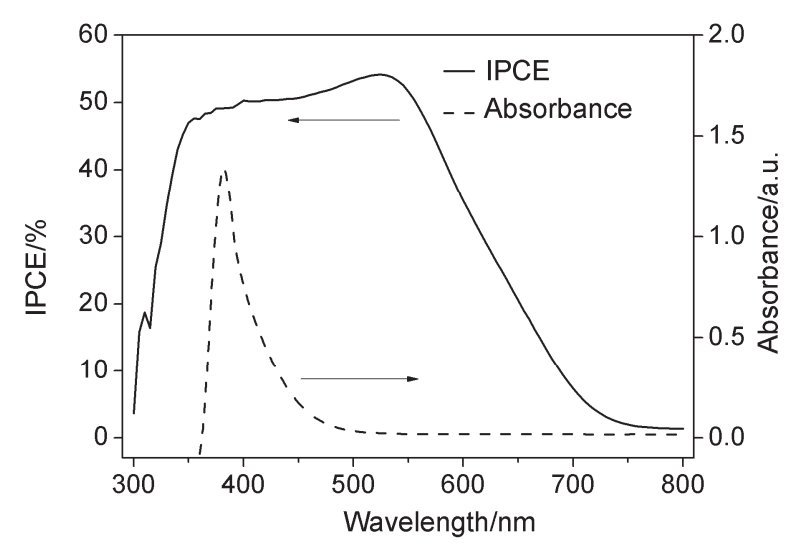

图 3 器件的 IPCE 响应与 DMcT 电解液的吸收谱

Figure 3 IPCE of the solar cell and absorption of DMcT electrolyte

\section{3 器件的循环伏安特性}

循环伏安曲线可以反映材料的电化学行为, 利用 $\mathrm{LiClO}_{4}$ 作为电解质, $\mathrm{DMF}$ 为溶剂, 利用铂盘作为工作电 极, 铂丝作为对电极, 甘录电极作为参比电极. 在 -0.8 到 $0.8 \mathrm{~V}$ 的范围内对材料的氧化峰与还原峰进行扫描, 循环伏安曲线如图 4 所示. 通过对 DMcT 的多次扫描, 可以看出其氧化还原特性相对稳定, 第 1 次循环与第 100 次循环相比, 氧化峰从 0.528 变为 $0.555 \mathrm{~V}$, 还原峰 从一 0.520 变为 $-0.577 \mathrm{~V}$, 峰位相对稳定, 在整个过程 中并没有出现较大的波动变化, 说明 DMcT 的电化学稳 定性较好, 有助于保证器件寿命的稳定. 而且通过多次 测试后的循环伏安曲线也可以看出, 见图 $\mathrm{S} 2, \mathrm{~T}^{-} / \mathrm{T}_{2}$ 的 氧化峰还原峰移动也要大于 DMcT. 但根据相关文献报 道, 电流的衰减主要是由于硫原子容易吸附在 $\mathrm{Pt}$ 表面, 从而降低 $\mathrm{Pt}$ 的催化活性, 导致器件在电解液/对电极界
面处的损耗较高 ${ }^{[13,22,23]}$. 因此在后续的工作中, 可以考 虑引入其他非 Pt 对电极材料, 以降低这种负面影响.

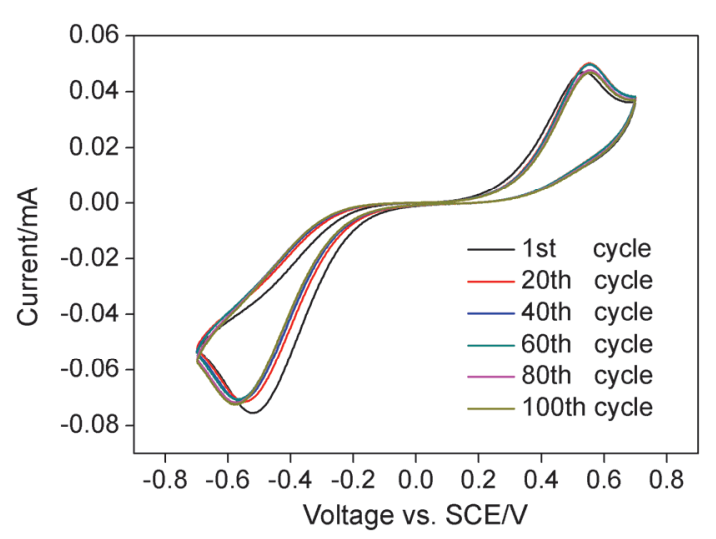

图 $4 \mathrm{DMcT}$ 的循环伏安特性

Figure 4 Cyclic-voltammetry curves of DMcT electrolyte

\section{4 器件的电化学阻抗谱}

通过循环伏安曲线的衰减可以发现 DMcT 的氧化 还原行为与对电极 $\mathrm{Pt}$ 密切相关, 为了更好地反映器件 中各个界面处的电荷转移行为, 可以通过电化学阻抗谱 来进行模拟分析. 分别以阻抗的实部和虚部可以做出 Nyquist 图，在染料敏化太阳能电池中，一般认为第一个 弧代表了电解液/对电极处的界面，第二个弧代表了光 阳极/电解液处的界面，通过适当的 $\mathrm{RC}$ 回路进行模拟， 可以推算出各个界面处的阻抗信息 ${ }^{[24 ~ 28]}$. 图 5 给出的是 将器件放置在暗态下, 并对器件施加 $V_{\mathrm{oc}}$ 大小的偏压进 行测试的结果. 通过拟合计算可得, $R_{\mathrm{CE}}$ 与 $R_{\mathrm{TiO} 2}$ 分别为 $221,202 \Omega \cdot \mathrm{cm}^{-2}$, 这可能是导致器件 $F F$ 较低的主要原 因 ${ }^{[26]}$. 较大的 $R_{\mathrm{CE}}$ 反映了 $\mathrm{Pt}$ 电极对于 $\mathrm{DMcT}$ 的催化活性 较低, 不利于电荷的快速传输, 这与循环伏安测量中发 现的结果也是一致的. 因此，后续工作应该主要围绕选 择适合于有硫化物的对电极展开, 如碳材料, 导电高分 子材料等. 此外 DMcT 的自均聚/自解聚反应中会形成 聚合物从而导致电解液粘度增加, 而粘度的增加会导致

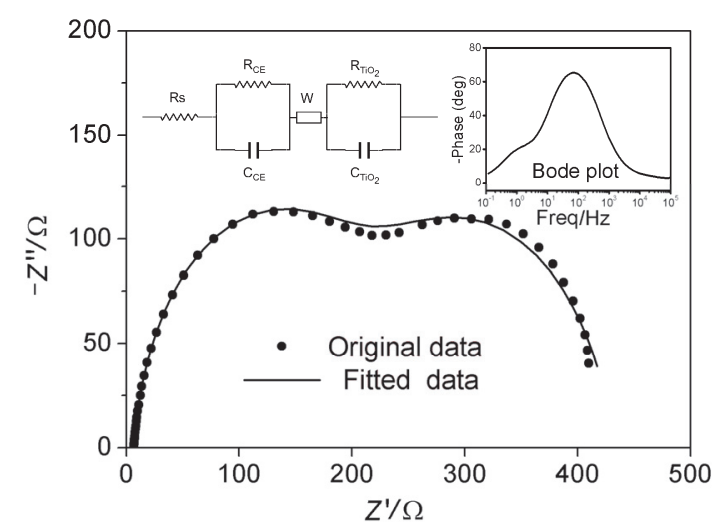

图 5 器件的电化学阻抗谱

Figure 5 Nyquist plots of the solar cell. The inset figure shows the equivalent model and Bode plots 
电解质的扩散系数降低, 不利于电荷的传输, 而且与其 它电解质体系相比, DMcT 器件的界面阻抗与系统阻抗 都比较高, 这些都会引起 FF 降低.

\section{3 结论}

总而言之, 基于自均聚/自解聚反应的新型氧化还 原电解质首次应用于染料敏化太阳能电池中. 经器件优 化后, 可以在标准光强下实现 $1.6 \%$ 的光电转化效率, 而 值得一提的是其在弱光 $\left(13 \mathrm{~mW} \cdot \mathrm{cm}^{-2}\right)$ 下的效率可以达 到 $2.6 \%$, 这意味着此类电池在室内等弱光条件下具有 较好的应用前景. 初步的实验结果表明, 器件的开路电 压和填充因子较低, 限制了效率的进一步提升. 而通过 循环伏安测试与电化学阻抗谱的结果来看, 金属铂电极 对于有机硫化物的氧化还原体系的催化活性并不高, 在 后续工作中可以尝试采用碳材料的对电极对此器件进 行优化. 虽然 DMcT 的效率还较低, 但是采用自均聚/自 解聚反应的氧化还原过程还是为开发新型氧化还原电 对提供了新的可能.

\section{4 实验部分}

\subsection{DMcT 电解液的配制}

按照以下浓度配制 DMcT 电解液, 其中 DMcT 的浓 度为 $0.2 \mathrm{~mol} / \mathrm{L}$, 其中的添加剂成分与浓度分别为 0.1 $\mathrm{mol} / \mathrm{L}$ 的 $\mathrm{LiClO}_{4}, 0.1 \mathrm{~mol} / \mathrm{L}$ 的 $\mathrm{GuSCN}$ 以及 $0.5 \mathrm{~mol} / \mathrm{L}$ 的 $\mathrm{TBP}$, 电解液溶剂采用 $\mathrm{DMAC} / \mathrm{ACN}$ 的混合溶剂, 混合 比例为 $1: 3$.

\section{2 工作电极与器件制备}

首先清洗 FTO 玻璃祄底, 将 FTO 玻璃分别浸泡在 去离子水、丙酮和无水乙醇中, 并各自超声清洗 $20 \mathrm{~min}$. 取出用氮气吹干, 之后放入氧等离子清洗器中, 清洗时 间 $15 \mathrm{~min}$, 然后分别制备光阳极与对电极. 光阳极的制 备方法如下: 将 $\mathrm{FTO}$ 玻璃浸入 $40 \mathrm{mmol} / \mathrm{L}$ 的 $\mathrm{TiCl}_{4}$ 水溶 液中, 保持在 $70{ }^{\circ} \mathrm{C}$ 下浸泡 $30 \mathrm{~min}$, 之后取出先用去离 子水和无水乙醇冲洗, 并用氮气吹干. 然后在上面生长 多孔 $\mathrm{TiO}_{2}$ 薄膜作为光阳极. 采用刮涂法在 FTO 玻璃上 涂布 $\mathrm{TiO}_{2}$ 浆料(其中 $\mathrm{TiO}_{2}$ 粒径约为 $20 \mathrm{~nm}$ ), 经过 $450{ }^{\circ} \mathrm{C}$ 高温加热烧结, 即可得到多孔 $\mathrm{TiO}_{2}$ 光阳极. 经测定, 多 孔薄膜层厚度约为 $12 \mu \mathrm{m}$. 将光阳极浸泡在配置好的染 料溶液中, 在常温下放置在暗处保持 $24 \mathrm{~h}$ 进行染料吸 附. 染料溶液配制方法如下: 将 $0.5 \mathrm{mmol} / \mathrm{L}$ 的 N719 染 料溶解在乙醇溶液中, 放置在暗处保持搅拌 $12 \mathrm{~h}$ 以上. 光阳极吸附染料后, 取出用无水乙醇冲洗, 并用氮气吹 干即可. 铂对电极的制备方法如下: 将清洗好的 FTO 玻 璃放入磁控溅射仪, 然后在表面沉积厚度约 $120 \mathrm{~nm}$ 的 薄膜即可. 最后将事先配好的 DMcT 电解液滴入光阳极
与对电极之间, 将光阳极、对电极与外电路相连进行各 项测试.

\section{References}

[1] O’Regan, B.; Grätzel, M. Nature 1991, 353, 737.

[2] Bach, U.; Lupo, D.; Comte, P.; Moser, J. E.; Weissortel, F.; Salbeck, J.; Spreitzer, H.; Grätzel, M. Nature 1998, 395, 583.

[3] Grätzel, M. Nature 2001, 414, 338.

[4] Hagfeldt, A.; Boschloo, G.; Sun, L.; Kloo, L.; Pettersson, H. Chem. Rev. 2010, 110, 6595 .

[5] Chiba, Y.; Islam, A.; Watanabe, Y.; Komiya, R.; Koide, N.; Han, L. Y. Jpn. J. Appl. Phys. 2006, 45, 638.

[6] Yuan, Q.; Chen, X. J.; Wang, J. T.; Zhai, J. Acta Chim. Sinica 2014 72, 624. (苑琪, 陈雪景, 王京涛, 翟锦, 化学学报, 2014, 72, 624.)

[7] Cao, Z. C.; He, Z.; Deng, L. J.; Tan, S. T. Chin. J. Org. Chem. 2014 34, 340. (曹镇财, 何舟, 邓利军, 谭松庭, 有机化学, 2014, 34, 340.)

[8] Wang, J. W.; Li, Y.; Xu, Y. L.; Li, Y.; Shen, K. H. Acta Chim. Sinica 2012, 70, 1278. (王纪伟, 李莹, 徐艳玲, 李杨, 申凯华, 化学 学报, 2012, 70, 1278.)

[9] He, J. J.; Chen, S. X.; Wang, T. T.; Zeng, H. P. Chin. J. Org. Chem. 2012, 32, 472. (何俊杰, 陈舒欣, 王婷婷, 曾和平, 有机化学, 2012, 32, 472.)

[10] Yum, J. H.; Baranoff, E.; Kessler, F.; Moehl, T.; Ahmad, S.; Bessho, T.; Marchioro, A.; Ghadiri, E.; Moser, J. E.; Yi, C.; Nazeeruddin, M. K.; Grätzel, M. Nat. Commun. 2012, 3, 631.

[11] Yella, A.; Lee, H. W.; Tsao, H. N.; Yi, C.; Chandiran, A. K.; Nazeeruddin, M. K.; Diau, E. W.; Yeh, C. Y.; Zakeeruddin, S. M.; Grätzel, M. Science 2011, 334, 629.

[12] Boschloo, G.; Hagfeldt, A. Acc. Chem. Res. 2009, 42, 1819.

[13] Wang, M. K.; Chamberland, N.; Breau, L.; Moser, J. E.; Humphry-Baker, R.; Marsan, B.; Zakeeruddin, S. M.; Grätzel, M. Nat. Chem. 2010, 2, 385.

[14] Tian, H. N.; Jiang, X. A.; Yu, Z.; Kloo, L.; Hagfeldt, A.; Sun, L. Angew. Chem., Int. Ed. 2010, 49, 7328.

[15] Li, D.; Li, H.; Luo, Y.; Li, K.; Meng, Q.; Armand, M.; Chen, L. Adv. Funct. Mater. 2010, 20, 3358.

[16] Tian, H. N.; Yu, Z.; Hagfeldt, A.; Kloo, L.; Sun, L. J. Am. Chem. Soc. 2011, 133, 9413.

[17] Daeneke, T.; Kwon, T. H.; Holmes, A. B.; Duffy, N. W.; Bach, U.; Spiccia, L. Nat. Chem. 2011, 3, 211.

[18] Bai, Y.; Yu, Q. J.; Cai, N.; Wang, Y. H.; Zhang, M.; Wang, P. Chem. Commun. 2011, 47, 4376.

[19] Liu, M.; Visco, S.; De Jonghe, L. C. J. Electrochem. Soc. 1991, 138 , 1896.

[20] Oyama, N.; Tatsuma, T.; Sato, T.; Sotomura, T. Nature 1995, 373, 598.

[21] Xiao, L. X.; Hou, T. L.; Guo, B. K.; Oyama, N. J. Central South Univ. (Sci. Technol.) 2004, 35, 774. (肖立新, 侯桃丽, 郭炳焜, 小 山昇, 中南大学学报 (自然科学版), 2004, 35, 774.)

[22] Wang, L.; Wu, M.; Gao, Y.; Ma, T. Appl. Phys. Lett. 2011, 98 221102.

[23] Wu, H.; Lv, Z.; Chu, Z.; Wang, D.; Hou, S.; Zou, D. J. Mater. Chem. 2011, 21, 14815 .

[24] Wang, M.; Moon, S. J.; Zhou, D.; Le Formal, F.; Cevey-Ha, N. L.; Humphry-Baker, R.; Grätzel, C.; Wang, P.; Zakeeruddin, S. M.; Grätzel, M. Adv. Funct. Mater. 2010, 20, 1821.

[25] Wang, Q.; Moser, J.-E.; Grätzel, M. J. Phys. Chem. B 2005, 109, 14945.

[26] Fabregat-Santiago, F.; Bisquert, J.; Cevey, L.; Chen, P.; Wang, M.; Zakeeruddin, S. M.; Grätzel, M. J. Am. Chem. Soc. 2009, 131, 558.

[27] Fabregat-Santiago, F.; Bisquert, J.; Palomares, E.; Otero, L.; Kuang, D.; Zakeeruddin, S. M.; Grätzel, M. J. Phys. Chem. C 2007, 111, 6550.

[28] Koh, J. K.; Kim, J.; Kim, B.; Kim, J. H.; Kim, E. Adv. Mater. 2011, $23,1641$.

[29] Fabregat-Santiago, F.; Bisquert, J.; Garcia-Belmonte, G.; Boschloo, G.; Hagfeldt, A. Sol. Energy Mater. Sol. Cells 2005, 87, 117. 